\title{
IGR Activity of Three Indigenous Plants belonging to Asteraceae Family and its Potential Role on Control of Filarial Vector, Culex quinquefasciatus Say (Diptera: Culicidae)
}

\author{
SR Ahalya', E Eushpalatha ${ }^{2}$
}

1,2Biopesticides \& Toxicology Division, Department of Zoology, University of Calicut, Malappuram, Kerala, India. DOI: https://doi.org/10.24321/0019.5138.202014

I $\quad \begin{array}{lllll}\mathbf{N} & \mathbf{F} & \mathbf{O}\end{array}$

Corresponding Author:

E Pushpalatha, Biopesticides \& Toxicology Division, Department of Zoology, University of

Calicut, Malappuram, Kerala, India.

E-mail Id:

drepushpalatha@gmail.com

Orcid Id:

https://orcid.org/0000-0002-1859-6338

How to cite this article:

Ahalya SR, Pushpalatha E. IGR Activity of Three Indigenous Plants belonging to Asteraceae Family and its Potential Role on Control of Filarial Vector, Culex quinquefasciatus Say (Diptera: Culicidae). J Commun Dis 2020; 52(2): 57-62.

Date of Submission: 2020-05-11

Date of Acceptance: 2020-06-28
$\begin{array}{lllllllll}\mathbf{A} & \mathbf{B} & \mathbf{S} & \mathbf{T} & \mathbf{R} & \mathbf{A} & \mathbf{C} & \mathbf{T}\end{array}$

Background: Insect Growth Regulatory and larvicidal role of three indigenous plants belonging to the family Asteraceae is tested against the filarial vector, Culex quinquefasciatus Say.

Methods: IGR and larvicidal bioassays were conducted using methanol and water extracts of the selected plants such as Blumea mollis, Vernonia cinerea and Centratherum punctatum against I instar larvae of $C x$. quinquefasciatus.

Result: Lethal concentrations $\left(\mathrm{LC}_{50}\right.$ and $\mathrm{LC}_{90}$ ) were estimated for methanol and aqueous extracts of the selected plants. Treatment with methanolic extracts exhibited prolonged larval and pupal duration, when compared to control. Total developmental period was also significantly extended. The larvae treated with aqueous extracts showed shortening of larval and pupal durations.

Conclusions: The present study highlights the importance of plantbased materials and their efficacy to control the mosquito vectors with special to Culex quinquefasciatus. Treatment with methanol extracts of the selected plants showed extension of larval duration where as aqueous extracts show precocenes like activity and the total developmental period shortened with the emergence of almost $90 \%$ of the treated larvae.

Keywords: IGR Activity, Asteraceae Plants, Filarial Vector, Culex quinquefasciatus, Blumea mollis, Vernonia cinerea and Centratherum punctatum, Larvicidal Activity

\section{Introduction}

Since mosquitoes are most abundant group of insect disease agents, control of them has become a major challenge to eradicate major epidemic diseases. Most of the developing countries have been suffering from frequent disease outbreaks. Several ways are in practice to control mosquitos like, physical, chemical, biological, and genetic ways. From 1920 onwards, chemical control 
became a predominant way of insect control. Due to the adverse effects of extensive use of chemical insecticides, the idea of biopesticides became more relevant. Since the effects of biopesticides are not persistent throughout years, it will not contribute to bio magnification and does not cause much development of resistance in organisms. The less toxic effects to non-target organisms is also an important feature of biopesticides. Since the sources of bio pesticides are plants and phytochemicals, they are easily biodegradable and eco-friendly. As the Integrated Vector Management (IVM) is the current strategy which includes a combination of all management techniques to control insect pests and vectors.

From the biopesticides point of view, phytochemicals do possess anti-insect properties which are either purely insecticidal or act as feeding deterrents, growth inhibitors, growth regulators, repellents or oviposition inhibitors against a variety of insect species. Biopesticides/ biolarvicides generally have several advantages compared to conventional pesticides ${ }^{1,2}$ as chemical pesticides are withdrawn owing to resistance problem, or because they are no longer commercially viable and, thus, immense opportunities are emerging for bio rational solutions.

IGRs are classified as (i) Juvenile hormone analogues (JHAs also called as Juvenoids) (ii) Ecdysteroid agonists and (iii) Chitin synthesis inhibitors ${ }^{3}$ (CSIs or moult-inhibitors). IGRs belong to a group of compounds which are not directly toxic, but selectively affect the growth and development, metamorphosis and reproduction of insects through the disruption of hormonally regulated physiological processes. ${ }^{4}$ Compounds with anti JH activities can be used as an insect control agent. The present study was carried out to assess insect growth regulatory and larvicidal properties of the three indigenous plants belonging to Asteraceae family and its potential role on control of filarial vector, Culex quinquefasciatus Say.

\section{Materials and Methods}

\section{Collection and Maintenance of Culex quinquefasciatus}

Larvae of Culex quinquefasciatus were collected from botanical garden of Calicut University and Kalluthan Kadavu colony, Kozhikode and brought to laboratory and reared. The adult mosquitoes were maintained at $29 \pm 2^{\circ} \mathrm{C}$ and $75 \pm 5 \%$ relative humidity at 12 -hour day and night cycle mimicking the natural condition in a 15 inch square cage covered with mesh. The adults were fed on $10 \%$ sucrose solution soaked in cotton and placed in a glass jar. Adequate blood meals were provided to 2 to 3 day old adult mosquitoes from an immobilized quail in twilight hours and a bowl containing water provided in the cage to facilitate oviposition. Egg rafts laid were transferred to a beaker and allowed to hatch. The freshly hatched larvae were fed by fine powder of yeast and dog biscuits in the ratio 3:1 and maintained in the laboratory.

\section{Preparation of Methanol Extracts}

The aerial parts of selected weed plants, Blumea mollis, Vernonia cinerea and Centratherum punctatum (Plate 1-3) were collected from the botanical garden of the University of Calicut, brought to the laboratory, washed thoroughly with water and shade dried at room temperature. The dried parts were powdered using a grinder and extracted in analytical grade methanol using a soxhlet apparatus. 15 to 20 cycles were fixed for each extract and collected separately and evaporated to dryness using a flash evaporator. The net weight of the extract was estimated and yield was calculated and $10 \%$ stock solution were prepared and stored in refrigerator for bioassay tests.

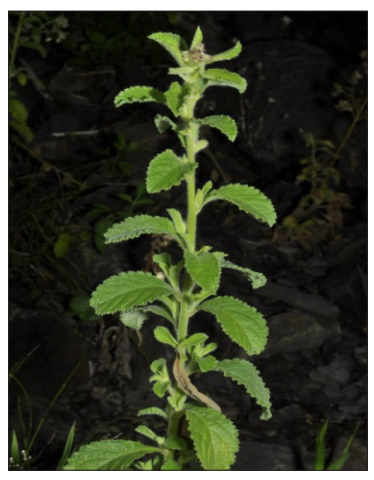

Plate I Blumea mollis (D.Don) Merr

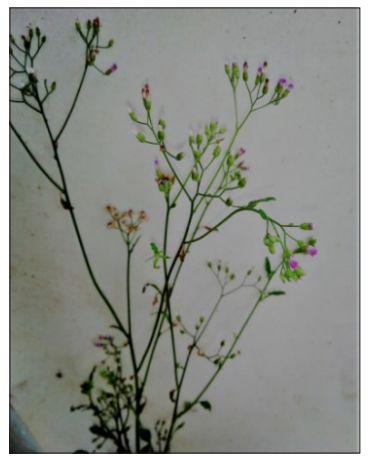

Plate 2 Vernonia cinerea (L)Less

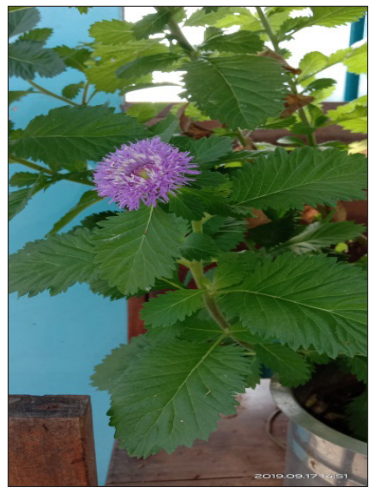

Plate 3 Centratherum punctatum Carr 


\section{Preparation of Aqueous Extracts}

$20 \mathrm{~g}$ plant powder mixed with $250 \mathrm{ml}$ distilled water in a conical flask was stirred for 24 hours in magnetic stirrer. The extract was then filtered and collected separately and evaporated to dryness. The yield of the plant material was calculated from the dried extract and $10 \%$ stock solution was prepared and stored in refrigerator.

\section{Bioassay}

\section{Estimation of Lethal Concentration $\mathrm{LC}_{50}$ and $\mathrm{LC}_{90}$}

Bioassay for the estimation of larvicidal activity using WHO protocol (1996) with slight modifications was adopted for the study. Methanol and water extracts of selected plants is tested against early first instar larvae of Culex quinquefasciatus. The appropriate volume of $1 \%$ stock is diluted to $100 \mathrm{ml} 0.08 \%$ saline water in a disposable glass to obtain the desired test medium. 10 larvae were released to each tumbler containing the test medium as $100 \mathrm{ml}$ test medium and also in the control set. Triplicates were maintained for each set. Observations on mortality of the larvae after $24 \mathrm{hr}$ was taken and percentage mortality was calculated. Using the percentage mortality, $\mathrm{LC}_{50}$ and $\mathrm{LC}_{90}$ was calculated using SPSS statistical programme.

\section{Insect Growth Regulatory Activity}

Insect growth regulating activity according to the protocol of Mehdi et $a{ }^{5}$ with slight modifications were conducted against freshly hatched 1 instar larvae of $C x$. quinquefasciatus. Fifty numbers of I instar larvae were transferred to a beaker containing $500 \mathrm{ml}$ of $0.08 \%$ saline solution. Desired sub lethal concentrations of the selected plant extracts were set to study the concentration depended IGR activity. Three replicates for experiment and controls were also maintained. Extension of total developmental duration, deformities and mortality rates were observed and recorded every day until adult emergence occurred. Larvae were provided with fine powder of dog biscuits and yeast in the ratio 3:1 respectively at fixed intervals during the observation period. Incidences of malformities were observed as larval-larval intermediates, larval-pupal intermediates and pupal-adult intermediates.

\section{Result}

Yield of methanol and aqueous extracts of selected plants, B. mollis, V. cinerea, and C. punctatum were estimated and provided in Table 1. Percentage yield of the methanol extracts of the selected plants ranged from $6.57 \%$ to $8.5 \%$ whereas that of aqueous extracts ranged from $5.79 \%$ to $9.21 \%$. Aqueous extract of $V$. cinerea shows highest percentage yield.

Table 2, provides $24 \mathrm{hrLC}_{50}$ and $\mathrm{LC}_{90}$ values of the methanol and aqueous extracts of $B$. mollis, $V$. cinerea, and $C$. punctatum. $24 \mathrm{hr} \mathrm{LC}$ ( $(\mathrm{ppm})$ of methanol extracts are $197.94 \mathrm{ppm}, 227.98 \mathrm{ppm}$ and $316.23 \mathrm{ppm}$ for C. punctatum, B. mollis, and $V$. cinerea respectively and that of aqueous extracts are $656.11 \mathrm{ppm}, 663.93 \mathrm{ppm}$, and $680.23 \mathrm{ppm}$ for B. mollis, V. cinerea, and C. punctatum, respectively. 24 $\mathrm{hr}$ LC90 values ranged from $410.9 \mathrm{ppm}$ to $556.7 \mathrm{ppm}$ for methanol extracts of $C$. punctatum and B. mollis and that of aqueous extract ranged from $701.9 \mathrm{ppm}$ to $872.7 \mathrm{ppm}$ for $B$. mollis and $C$. punctatum respectively.

Table I.Percentage yield obtained for the different extracts of B. mollis, V. cinerea, and C. punctatum

\begin{tabular}{|c|c|c|c|c|}
\hline S. No. & Name of the Plant & Material used for Extraction & Solvent used for Extraction & \% Yield \\
\hline \multirow{2}{*}{1.} & \multirow{2}{*}{ Blumea mollis } & \multirow{2}{*}{ whole areal parts } & Methanol & $7.69 \%$ \\
\hline & & & Aqueous & $5.79 \%$ \\
\hline \multirow{2}{*}{2.} & \multirow{2}{*}{ Vernonia cinerea } & \multirow{2}{*}{ whole areal parts } & Methanol & $8.50 \%$ \\
\hline & & & Aqueous & $9.21 \%$ \\
\hline \multirow{2}{*}{3.} & \multirow{2}{*}{ Centratherum punctatum } & \multirow{2}{*}{ whole areal parts } & Methanol & $6.57 \%$ \\
\hline & & & Aqueous & $7.40 \%$ \\
\hline
\end{tabular}

Table 2.24 hrs LC ${ }_{50}$ and LC 90 (ppm) and related statistics of the methanol and aqueous extracts of the selected plant extracts tested against first instar larvae of $C_{x}$. quinquefasciatus

\begin{tabular}{|c|c|c|c|c|}
\hline Plant & Extract & $\mathbf{L C}_{50}$ (UL-LL) & LC $_{90}$ (UL-LL) & Significance \\
\hline \multirow{3}{*}{ B. mollis } & Methanol & $227.98(287.57-155.62)$ & $556.72(1706.49-398.98)$ & 0.001 \\
\cline { 2 - 5 } & Aqueous & $656.11(651.55-561.15)$ & $701.91(691.70-617.42)$ & 0.000 \\
\hline \multirow{3}{*}{ V. cinera } & Methanol & $316.23(379.65-273.2)$ & $495.9(811.4-404.7)$ & 0.000 \\
\cline { 2 - 5 } C.punctatum & Aqueous & $663.93(674.29-603.96)$ & $743.180(820.66-701.73)$ & 0.018 \\
\cline { 2 - 5 } & Methanol & $197.94(278.9-32.32)$ & $410.92(352,24-289.95)$ & 0.001 \\
\cline { 2 - 5 } & Aqueous & $680.23(636.27-576.78)$ & $872.73(2178.4-768.26)$ & 0.005 \\
\hline
\end{tabular}




\section{Insect Growth Regulatory Activity}

\section{Effect of Methanolic Extract on Developmental duration of $C x$. quinquefasciatus}

During developmental progress, the larval and pupal periods were observed and compared with that of control. Freshly hatched I instar larvae treated with methanolic extract, the larval duration and pupal duration showed increase resulting in extension of the total life span of the treated Culex quinquefasciatus (Figure 1). Methanol extracts of all the three selected plants showed extended duration of the larval and pupal periods thereby extending the total developmental period for the three different concentrations tested when compared to that of control. Treatment of first instar of $C_{X}$. quinquefasciatus with $114 \mathrm{ppm}$ of the methanol extract of $B$. mollis shows an extension of developmental period up to $23.5 \pm 1.2$ days. Methanol extract of $V$. cinerea shows an extension of developmental period up to $24.0 \pm 1.0$ days than that of control ( $12.0 \pm 0.0$ days). A maximum of extended developmental period of $25.5 \pm 0.5$ days was observed when the first instar of $C x$. quinquefasciatus was treated with $85 \mathrm{ppm}$ of the methanol extract of $C$. punctatum (Figure 1).

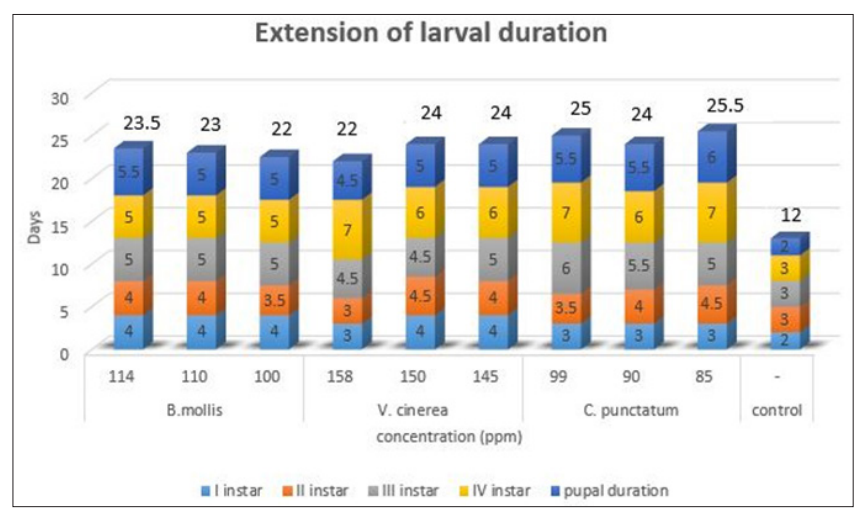

Figure I.Effect of Methanol extract of selected plants on the developmental duration of Culex quinquefasciatus

\section{Effect of Aqueous Extracts on Developmental duration of $C x$. quinquefasciatus}

Effect on the total developmental duration of $C x$ quinquefasciatus when treatment with aqueous extracts of the three selected plants were observed, recorded, and presented (Figure 2). When the freshly hatched first instar larvae were treated with aqueous extracts, it showed a decrease in total developmental duration when compared to that of control. The aqueous extracts induced shortening of larval-larval period, larval-pupal period, and pupal-adult period (Figure 2). Treatment with $278 \mathrm{ppm}$ of aqueous extract of $B$. mollis completes its developmental period within $10.0 \pm 0.0$ days. Total developmental period was decreased to $8.0 \pm 0.0$ days when treatment with $282 \mathrm{ppm}$ aqueous extract of $V$. cinereal whereas it was decreased to $7.5 \pm 0.5$ when treated with $285 \mathrm{ppm}$ aqueous extract of C. punctatum (Figure 2) when compared to that of control (11.5 \pm 0.5 days).

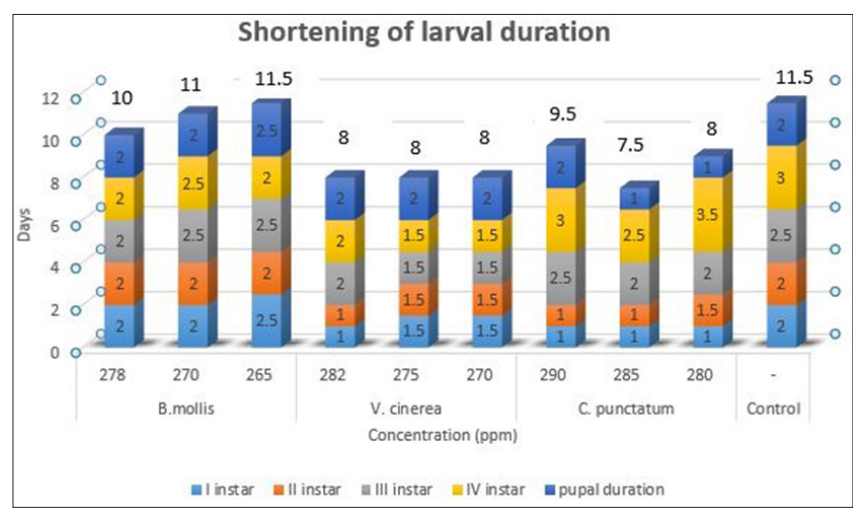

Figure 2.Effect of Aqueous Extract of the selected plants on the developmental duration of Culex quinquefasciatus

An exponential growth observed with increase in concentration and larvae treated with aqueous extracts showed shortening of larval and pupal periods. Total developmental period also shortened and allowed with almost above $90 \%$ emergence (Figure 3 ).

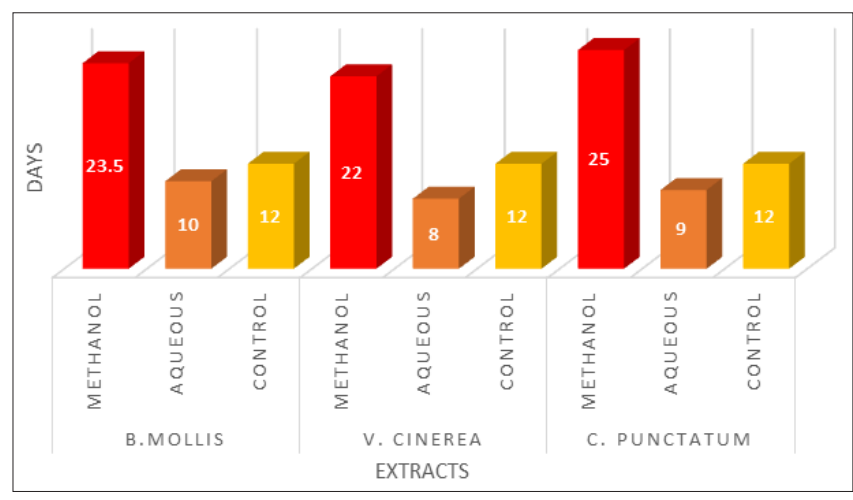

Figure 3.Effect of methanol and aqueous extracts of the selected plants on the total developmental duration of $C x$. quinquefasciatus

\section{Discussion}

Although several control measures for mosquito vectors have been practiced, the efforts are futile mainly due to the development of resistance against the synthetic insecticides. Insect Growth regulators (IGRs) and Chemosterilants offer considerable potential for the control of insect pests/ vectors. ${ }^{6}$ IGRs have low mammalian toxicity and do not appear to cause spontaneous genetic mutation and these materials are relatively short lived in the environment which reduces potential of contamination. Azardirachtin is the most widely used botanical insect growth regulator. ${ }^{7}$ Precocene, a plant product, is noted for its unique action interfering with growth by transgressing certain stages of 
development. In mosquitoes, it prevents pupal formation and adult emergence when newly hatched young larvae are exposed. ${ }^{8}$ Despite the toxic effects and reduction in emergence rate, insect growth regulators also exhibited combined effects on the developmental period and adult emergence with extending to the developmental duration of exposed larvae. ${ }^{9}$ The plant extracts caused larval mortality in a dose-dependent manner. All crude methanol extracts have been found to possess promising larvicidal activity with $\mathrm{LC}_{50}$ values which is on par with several reported works.

Present study also provides the proof of IGR activity induced by the plant extracts on $C x$. quinquefasciatus based on the developmental process. Treatment with methanolic extracts exhibited prolonged larval and pupal periods, when compared to control. Total developmental period was also significantly extended. On the other hand, on treatment with aqueous extracts of selected plants, larvae showed enormous growth and completed their life cycle within short period of time.

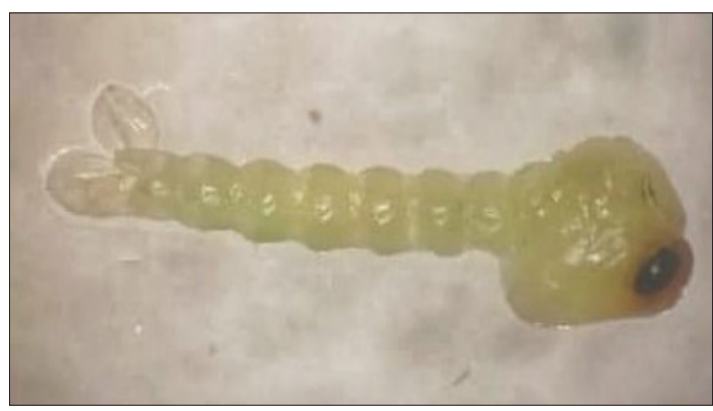

Figure 4.Larval-pupal Intermediate

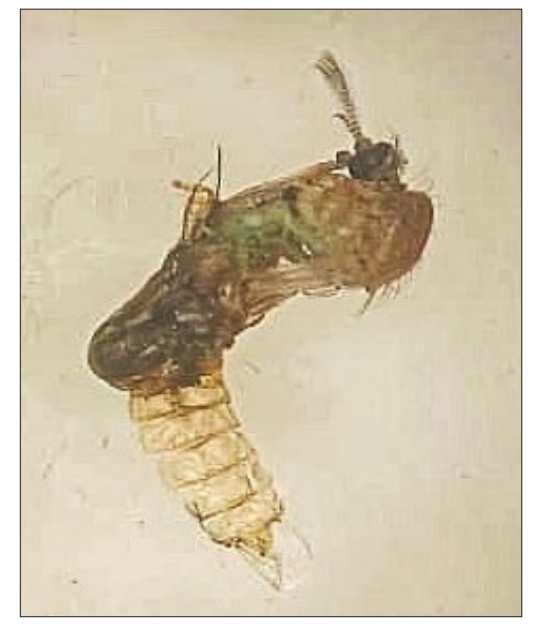

Figure 5.Pupal-adult intermediate

Furthermore, the developmental progress was affected by showing several deformities including moult inhibition, morphological abnormalities and mortality especially during moulting and melanisation processes. All the methanolic extracts attributed to the dechitinizing effect of extracts with the formation of larval-larval intermediates, larvalpupal intermediates, and pupal-adult intermediates. In the case of larval-larval intermediates, death has occurred with moulting sutures on head of the larvae, therefore it could not moult into next instars whereas, the formation of larvalpupal intermediates resulted in death of treated larvae at an early stage of pupation. The abdomen has also retracted along the larval skin and adopted the characteristic of pupal shape. The pupal abdomen has stretched like larvae with pupal head (Figure 4). Whereas, in the case of pupal-adult intermediates, death occurred after complete moulting, but some parts remained attached with pupal case (Figure 5).

So, in larval-pupal intermediates, the moulted pupae could not attain the pupal characteristics such as coma shape or curved abdomen, but it appeared with stretched abdomen, probably due to the presence of JH in pupae. Similarly, in the case of pupal-adult intermediates, the adult which was emerged from the pupae existed with pupal exuvia, which might to be due to the presence of increasing amount of $\mathrm{JH}$ and decreasing amount of ecdysone on the adult mosquitoes, as only the complete absence of $\mathrm{JH}$ results in formation of the adults. ${ }^{10}$

\section{Conclusion}

Methanol and aqueous extracts of the three indigenous plants, namely Blumea mollis, Vernonia cinerea and Centratherum punctatum belonging to the family Asteraceae shown its potential role of larvicidal and insect growth regulatory activities against $C x$. quinquefasciatus Say. During the developmental metamorphosis, treated larvae exhibited extension of total developmental duration, such as prolonged larval and pupal duration in methanol extracts and shortened larval and pupal period in aqueous extracts. Therefore, the present study suggests that these plant materials can moderately control the population of filarial vector mosquito, $C x$. quinquefasciatus Say and hence can be used as natural, ecofriendly and bio-degradable material for the control of mosquito vectors. However, isolation of the active principles of the selected plant extracts of the present study and its formulations is progressing which may lead to a better alternative to the synthetic larvicides for the control of mosquito vectors.

\section{Acknowledgement}

Authors acknowledge UGC, New Delhi for the technical support provided through MRP. Authors are thankful to Department of Zoology, University of Calicut, Malappuram, Kerala for providing laboratory facilities.

Conflict of Interest: None

\section{References}

1. Kaya HK, Lacey LA. Introduction to microbial control. In: Lacey, L.A, Kaya, H.K. (Eds), Field manual of techniques in Invertebrate Pathology: Application and Evaluation of Pathogens for control of Insect and other 
invertebrate pests, Second Edn. Springer, Dordrecht, The Netherlands. 2007; 3-7.

2. Kaya HK, Vega FE. Scope and Basic principles of Insect pathology. In: Vega, F., Kaya, H.K., Editors. Insect Pathology $2^{\text {nd }} E d n$. San Diego, CA: Academic Press. 2012; 1-12.

3. Oberlander H, Silhacek DL. Insect Growth Regulators, In: Alternatives to pesticides in stored product IPM (eds. B. Subramanyam and D.W. Hagstrum). Kluwer Academic Publishers, Boston. 2000; 147-163.

4. Wang QL, Liu T-X. Effects of three insect growth regulators on Encarsia formosa (Hymenoptera: Aphelinidae), an endoparasitoid of Bemisia tabaci (Hemiptera: Aleyrodidae). Journal of Economic Entomology 2016; 109(6): 2290-2297. DOI: 10.1093/ jee/tow216.

5. Mehdi SH, Qamar A, Khan I, Jacob P. Larvicidal And IGR Potential of Ocimum tenuiflorum and Datura alba leaf extracts against Malaria Vector. European Journal of Experimental Biology 2012; 2(4): 1370-1375.

6. Arivoli S, Tennyson S. Larvicidal and adult emergence inhibition activity of Abutilon indicum (Linn.) (Malvaceaea) leaf extracts against vector mosquitoes (Diptera : Culicidae). Journal of Biopesticides 2011; 4 (1): 27-35.

7. Hoffmann KH, Lorenz MW. Recent advances in hormones in insect pest control. Phytoparasitica 1998; 26(4): 323-330.

8. Cupp EW, Lok JB, Bowers WS. The developmental effects of 6,7 dimethoxy 2,2 dimethyl chromene on the pre-imaginal stages of Aedes aegypti (Diptera: Culicidae). Entomologis Experimentalis et Applicata 1977; 22(1): 23-28.

9. Kamalakannan S, Murugan K, Chandramohan B. Insect growth regulatory activity of Acalypha alnifolia (Euphorbiaceae) and Vitex negundo (Verbenaceae) leaf extracts against Aedes aegypti (Diptera: Culicidae). International Journal of Mosquito Research 2015; 2(1): 47-52.

10. Wigglesworth VB. Memoirs: The physiology of ecdysis in Rhodinus prolixus (Hemiptera). II Factors controlling moulting and metamorphosis. Journal of Cell Science 1934; 2(306): 191-222. 